\title{
Tacrolimus nanoparticles based on chitosan combined with nicotinamide: enhancing percutaneous delivery and treatment efficacy for atopic dermatitis and reducing dose
}

This article was published in the following Dove Press journal:

International Journal of Nanomedicine

\section{Kaiyue Yu \\ Yixuan Wang \\ Tao Wan \\ Yuanhao Zhai \\ Sisi Cao \\ Wenyi Ruan \\ Chuanbin Wu \\ Yuehong $\mathrm{Xu}$}

School of Pharmaceutical Sciences, Sun Yat-sen University, Guangzhou, People's Republic of China
Correspondence: Yuehong Xu School of Pharmaceutical Sciences, Sun Yat-sen University, Number 132, Out-ring East Road, University Town, Guangzhou 510006, People's Republic of China

Tel/fax +86 2039943119

Email Issxyh@mail.sysu.edu.cn
Background: Topical application of tacrolimus (FK506) was effective in the treatment of atopic dermatitis (AD); however, adverse effects frequently occurred with the increase of FK506 dose during long-term treatment.

Objective: The objective of this project was to develop a hybrid skin targeting system encapsulating FK506 based on nicotinamide (NIC) and chitosan nanoparticles (CS-NPs), ie, FK506NIC-CS-NPs, which took advantages of both of NIC and CS-NPs to obtain the synergetic effects of percutaneous delivery and treatment efficacy enhancement along with dose reduction.

Methods: The formulation of FK506-NIC-CS-NPs was optimized and characterized. In vitro and in vivo skin permeation studies were performed. AD-like skin lesions were constructed with BALB/c mice by 1-chloro-2, 4-dinitrobenzene (DNCB)-induced, and FK506-NIC-CS-NPs containing different dose of FK506 were topically administered to treat AD-like skin lesions in comparison with Protopic.

Results: NIC was found to significantly increase the FK506 EE to $92.2 \%$ by CS-NPs. In comparison with commercial FK506 ointment (Protopic), in vitro and in vivo skin permeation studies demonstrated that NIC-CS-NPs system significantly enhanced FK506 permeation through and into the skin, and deposited more FK506 into the skin. The treatment efficacy on clinical symptoms, histological analysis, and molecular biology of the AD-mice demonstrated that NIC-CS-NPs with 1/3 dose of FK506 of Protopic was superior to that of Protopic, and NIC-CS-NPs vehicle exhibited the adjuvant therapy and moderate anti-AD effects.

Conclusion: The system of NIC-CS-NPs enhances the permeability of FK506, plays an adjuvant role in anti-AD, reduces the dose of FK506 in treating $\mathrm{AD}$, and is therefore a promising nanoscale system of FK506 for the effective treatment of AD.

Keywords: tacrolimus, chitosan, nanoparticles, nicotinamide, atopic dermatitis, percutaneous delivery, reducing dose

\section{Introduction}

Atopic dermatitis (AD) is a chronically relapsing inflammatory skin disease with severe pruritus and eczema that occurs in adults but is encountered most frequently in children, with an increasing prevalence in infants and children worldwide. ${ }^{1}$ The clinical presentation of $\mathrm{AD}$ is characterized by pruritic, crusted, and erythematous skin lesions; these symptoms impair the quality of life of patients and affect their social lives. ${ }^{2,3}$ Mechanisms of $\mathrm{AD}$ are complex, by which elevated levels of immunoglobulin $\mathrm{E}$ (IgE), T helper 2 (Th2) cell recruitment, and production of cytokines such as interleukin (IL)-4, IL-5, and 
IL-13 occur, but generally start with dysfunction of the skin barrier. ${ }^{4}$ Topical application of corticosteroids is the first-line therapeutic option for the treatment of AD. However, AD, a chronic skin disease, often requires long-term and routine administration of corticosteroids that frequently leads to significant adverse effects such as skin atrophy, telangiectasia, striae, hyper- and hypopigmentation, and tachyphylaxis. 5,6

Tacrolimus (FK506), a potent macrolide immunosuppressive agent, has been proved to be notably effective in the treatment of immune-mediated cutaneous disorders including AD. ${ }^{7-9}$ Protopic (Astellas Toyama Co., Ltd, Toyama, Japan), the commercially available topical ointment formulation of FK506, has been approved by the FDA for the treatment of AD with two concentrations: $0.1 \%(\mathrm{w} / \mathrm{w})$ for adults over age 16 and $0.03 \%(\mathrm{w} / \mathrm{w})$ for children over age 2 . It has become an alternative treatment of $\mathrm{AD}$ and is indicated for use on the face and neck; ${ }^{10}$ the efficacy of the $0.1 \%$ (w/w) FK506 ointment for $\mathrm{AD}$ is almost equivalent to that of a strong-class corticosteroid based on randomized controlled clinical trials, ${ }^{11-13}$ and it was the preferred long-term treatment of $\mathrm{AD}$ to prevent disruption of the skin barrier. ${ }^{14}$ FK506 immunomodulates various skin disorders through entering $\mathrm{T}$ cells and blocking calmodulin, thus resulting in inhibition of T-cell expression, ${ }^{15}$ whereas T-cell activation is important for the development and persistence of AD. Moreover, FK506 can bind to steroid receptors on the cell surface, inhibit the release of histamine and serotonin from mast cells, and inactivate Langerhans cells. ${ }^{16,17}$ Although topical application of FK506 has not produced significant adverse effects as with corticosteroids, the transient burning sensation and hot flashes at the application site often lead to withdrawal from therapy with FK506 ointment; moreover, it has been reported that when patients are treated with the FK506 ointment with AD for 3 weeks, the burning sensation became more frequent with the increase in the FK506 dose from $0.03 \%$ to $0.3 \%$ (w/w). ${ }^{18}$ Moreover, when applied for 12 weeks in the treatment of AD, systemic adverse effects including flu-like symptoms, erythema, and headaches occurred. ${ }^{19}$ It is clinically worthwhile to formulate a drug-delivery system to enhance FK506 permeation into the skin and thus reduce its topically applied dose to minimize the occurrence of adverse effects and to maintain efficacy at the same time. The drug-delivery system with the capacity of depositing more drug and persisting in the skin is expected to exhibit skin-targeting effects. The high molecular weight (MW 822.05 Da), high hydrophobicity ( $\log$ P 6.09), and poor water solubility (4-12 $\mu \mathrm{g} / \mathrm{mL})$ of FK506 can limit its ability to effectively permeate into the skin. ${ }^{20}$ In previous studies, small amounts of FK506 from Protopic was deposited in the skin. ${ }^{21-23}$ With the aim to ensure that sufficient drug permeated into the skin from ointment to reach the therapeutic window of FK506, Protopic is required to be administered in higher quantities, with consequences such as increasing side effects and premature discontinuation. Thus, it is probably feasible to formulate a percutaneous drug-delivery system that enhances more FK506 permeation into the skin than Protopic, and consequently reduces FK506 dose for treating AD.

Various formulations based on nano-sized carriers included microemulsion, ethosomes, lipid nanoparticles, modified nanolipid carrier, and polymer micelle nanocarriers have been proven to be efficient approaches to improve percutaneous delivery of FK506. ${ }^{22,24-30}$ These nanocarriers facilitate drug delivery to structural features of the skin, such as hair follicles, or interact with skin lipids to mediate transportation and create a drug depot in the skin for sustained release. . $^{31,32}$ Moreover, cationic nanoparticles have shown advantages of enhancing drug permeation over neutral or negatively charged nanoparticles as drug nanocarriers; significant progress has been made in cationic nanoparticles for drug delivery. ${ }^{33,34}$ In the present study, chitosan nanoparticles (CS-NPs) were used as the carrier of FK506 for percutaneous delivery. CS is a cationic polysaccharide with favorable biological characteristics such as biodegradability, biocompatibility, and nontoxicity. ${ }^{35}$ It has been widely applied to construct NPs and applied for topical skin delivery. ${ }^{36}$ Compared to the neutral or negatively charged NPs, CS-NPs with positive charges from amino groups $\left(\mathrm{NH}^{3+}\right)$ of $\mathrm{CS}$ can bind to the epithelial cell membrane, depolymerize F-actin, and disband the tightjunction protein ZO-1, resulting in opening of tight junctions in the skin and enhancing the percutaneous permeation of the drug. ${ }^{37}$ Furthermore, CS works as an antibacterial agent through interaction with negatively charged surface components of numerous bacteria, and derives additional wound-healing benefits when it is used as a vehicle of skin pharmaceuticals. ${ }^{38,39}$ With these attributes, CS is a desirable polymer for constructing NPs for delivering therapeutics to treat skin diseases. However, the hydrophilic nature of CS has limited its use to encapsulating highly hydrophobic drugs. ${ }^{40}$ Therefore, with the aim to increase the loading capacity of CS-NPs for highly hydrophobic FK506, the hydrotropic agent nicotinamide (NIC) was introduced.

NIC, a water-soluble derivative of vitamin $B_{3}$, is a hydrotropic agent and has shown the ability to enhance the solubility of some poorly water-soluble molecules. ${ }^{41-44}$ Our previous study has demonstrated that NIC solubilizes FK506 through forming an FK506-NIC complex and enhances FK506 permeation into the skin. ${ }^{21}$ NIC has been used as a 
cosmetic active ingredient for its moisturizing and depigmenting properties, and is a cheap generic drug with a wellestablished safety profile in humans. It has been reported to be an inhibitor of PARP-1 that controls NF- $\kappa$ B-mediated transcription and, therefore, regulates the expression of adhesion molecules, pro-inflammatory mediators, and inflammatory cytokines, including IL-1, IL-13, and TNF- $\alpha$, that play key roles in the pathogenesis of AD. ${ }^{45,46}$ In this study, FK506NIC-CS-NPs were prepared for the first time by using NIC to overcome the high hydrophobic property of FK506 and increase its loading into CS-NPs, and we hypothesize that FK506-NIC-CS-NPs can take advantage of both CS-NPs and NIC, including solubilizing FK506, enhancing percutaneous penetration of FK506, and synergizing FK506 for treating $\mathrm{AD}$, thus finally reducing the FK506 dose to minimize adverse effects while maintaining its efficacy.

Based on the abovementioned considerations, FK506NIC-CS-NPs were developed and characterized. In vitro skin permeation was undertaken to investigate the enhancing effect of NIC-CS-NPs on FK506 penetration into and through rat skin, and in vivo skin permeation was undertaken to further understand the distribution of FK506 in different skin layers. $\mathrm{AD}$-like skin lesions in $\mathrm{BALB} / \mathrm{c}$ mice were induced by 1-chloro-2,4-dinitrobenzene (DNCB), and treatment effects on clinical symptoms, histological analysis, and molecular biology of AD mice were comparatively studied between FK506-NIC-CS-NPs, containing different FK506 doses, and Protopic, with 0.1\% (w/w) FK506.

\section{Materials and methods Materials}

Chitosan with a deacetylation degree of $75.0 \%-85.0 \%$ $\left(5-20 \mathrm{mPa} \cdot \mathrm{s}, 0.5 \%\right.$ in $0.5 \%$ acetic acid at $20^{\circ} \mathrm{C}$ ) was obtained from Tokyo Chemical Industry (Tokyo, Japan). Sodium tripolyphosphate (TPP) was purchased from Aladdin (Shanghai, People's Republic of China). NIC was a gift of Guangzhou Changlong (Guangzhou, People's Republic of China). FK506 was purchased from Teva Czech Industries (S.R.O Ostravska 29305, 74770 Opava-Komarov). The FK506 ointment (Protopic ${ }^{\circledR}$ ) was obtained from Astellas Toyama Co., Ltd. (Toyama, Japan). DNCB was purchased from Sigma-Aldrich (St Louis, MO, USA). Other reagents were of analytical grade.

Sprague Dawley rats (male, 180-220 g) and BALB/c mice (male, 4 weeks) were obtained from the Experimental Animal Center of Sun Yat-sen University (Guangzhou, People's Republic of China). Animals were housed under specific pathogen-free conditions at constant levels of humidity and temperature on 12-h light/dark cycles, and provided with food and water ad libitum. All work undertaken with animals were in accordance with the Principles of Laboratory Animal Care and Use in Research published by the Chinese Ministry of Health, and the protocols [SCXK (YUE) 2016-0029] were approved by the Institutional Animal Care and Use Committee of Sun Yat-sen University.

\section{Formulation of NPs \\ Preparation of blank CS-NPs}

The CS-NPs were prepared via the ionic gelation method with TPP as a crosslinker. ${ }^{47,48}$ Briefly, CS was dissolved in $1 \%(\mathrm{v} / \mathrm{v})$ acetic acid solution under continuous stirring for $4 \mathrm{~h}$ to obtain a CS solution of $2.0 \mathrm{mg} / \mathrm{mL}$ with a $\mathrm{pH}$ value of 5.0 adjusted by $2 \mathrm{M}$ sodium hydroxide solution. The TPP solution was prepared at a concentration of $8.0 \mathrm{mg} / \mathrm{mL}$. CS-NPs were obtained upon the addition of $100 \mu \mathrm{L}$ TPP solution to $2 \mathrm{~mL} \mathrm{CS}$ solution with a weight ratio of CS/TPP at 5/1 under magnetic stirring at $500 \mathrm{rpm}$ for $30 \mathrm{~min}$.

\section{Preparation of FK506-NIC complex solution}

Excess FK506 was dispersed in 10\%, 20\%, and 40\% (w/v) NIC aqueous solutions, respectively, and vibrated for $24 \mathrm{~h}$. The supernatant was filtered through a membrane filter $(0.22 \mu \mathrm{m})$ to obtain the FK506-NIC complex solution, and the FK506 concentration in the complex solution was determined with high-performance liquid chromatography (HPLC).

\section{Preparation of FK506-NIC-CS-NPs}

Chitosan solutions were prepared as in the section "Preparation of blank CS-NPs", with different pHs of 4.5, 5.0, and 5.5 , respectively. Two milliliters of FK506-NIC complex solution containing 40\% (w/v) NIC and 0.2\% (w/v) FK506 was added into $2 \mathrm{~mL} C S$ solution with different $\mathrm{pHs}$, respectively; then, $100 \mu \mathrm{L}$ TPP solution was added into the mixture for ion gelation under magnetic stirring at $500 \mathrm{rpm}$ for 30 min to obtain FK506-NIC-CS-NPs containing 20\% $(\mathrm{w} / \mathrm{v}) \mathrm{NIC}$ and $0.1 \%(\mathrm{w} / \mathrm{v})$ FK506. The resultant $\mathrm{pH}$ value of the FK506-NIC-CS-NPs suspension was 5.0, 5.4, and 5.9, respectively. The NIC-CS-NPs suspension was prepared according to the same process, but without FK506. Moreover, $2 \mathrm{~mL}$ FK506-NIC complex solution containing 40\% (w/v) NIC and $0.02 \%, 0.06 \%$, and $0.1 \%$ (w/v) FK506 was added into $2 \mathrm{~mL} \mathrm{CS}$ solution at pH 5.0 to obtain FK506-NIC-CSNPs with different FK506 loading concentrations of $0.01 \%$, $0.03 \%$, and $0.05 \%(\mathrm{w} / \mathrm{v})$. 


\section{Characterization of NPs}

Size distribution and zeta potential (ZP) of NPs were characterized by dynamic light scattering (DLS; Mavlvern Zetasizer Nano-ZS90, Worcestershire, UK). Aqueous suspension of NPs diluted 10 -fold by deionized water were placed into DTS0012 and DTS1070 cuvettes, respectively, to measure the size, polydispersity index (PDI), and $\mathrm{ZP}$ at $25^{\circ} \mathrm{C}$.

The FK506 encapsulation efficiency (EE, \%) of NPs was evaluated with gel column chromatography. NPs and unloaded FK506 were separated through the Sephadex LH-20 column, FK506 was determined with HPLC, and the EE was calculated as follows:

$$
\text { EE }(\%)=\frac{\begin{array}{c}
\text { Total amount of FK } 506 \\
\text { Amount of unloaded FK506 }
\end{array}}{\text { Total amount of FK506 }} \times 100 \%
$$

\section{Skin permeation of FK506-NIC-CS-NPs in vitro}

The Sprague Dawley rats were anesthetized and sacrificed by cervical dislocation, and the hair on the abdomen was carefully shaved off with a razor. The abdominal skin was excised, the subcutaneous tissues were removed surgically and rinsed with normal saline (NS), and, then, the skin samples were stored at $-20^{\circ} \mathrm{C}$ until use. In vitro permeation was undertaken with a Franz diffusion cell under occlusive conditions, with an effective diffusion area of $3.14 \mathrm{~cm}^{2}$ and receptor volume of $7.5 \mathrm{~mL}$ (TK-12A, Kaikai Technology., Ltd, Shanghai, People's Republic of China). The skin was mounted between the donor and receiver compartments with the stratum corneum (SC) facing the donor compartment. Each receiver compartment was filled with $7.5 \mathrm{~mL} \mathrm{NS}$ containing $25 \%$ (v/v) ethanol as the receiving medium with respect to the sink conditions ascertained per our previous study, ${ }^{21}$ stirred at $300 \mathrm{rpm}$, and maintained at $37^{\circ} \mathrm{C} \pm 0.5^{\circ} \mathrm{C}$ in a circulating water bath. Then, $0.5 \mathrm{~g}$ FK506-NIC complex solution, FK506-NIC-CS-NPs [containing an equal amount of NIC of $20 \%$ (w/v) with the FK506-NIC complex solution], FK506 suspension, and commercial preparation of FK506 (Protopic) containing the same content of FK506 (0.1\%, $\mathrm{w} / \mathrm{v})$ were placed into the donor compartment, respectively ( $\mathrm{n}=6$ for each group). At predetermined time intervals (12, $14,16,20$, and $24 \mathrm{~h}$ ), $1 \mathrm{~mL}$ sample was collected from the receiver compartment and equal amounts of fresh receiving medium was supplemented immediately. At the end of the in vitro permeation study, the skin demounted from the Franz cell was cleaned, cut into small pieces, and placed in a centrifuge tube. One milliliter methanol was used to extract
FK506 retained in the skin slices by sonicating for $30 \mathrm{~min}$ and then centrifuged at 5,000 rpm for $5 \mathrm{~min}$. The supernatant was collected and filtered with a $0.22-\mu \mathrm{m}$ microporous nylon membrane. FK506 concentration in each sample was determined with HPLC.

\section{Skin permeation of FK506-NIC-CS-NPs in vivo}

For in vivo permeation, 24 Sprague Dawley rats were anesthetized with urethane $(20 \%, \mathrm{w} / \mathrm{v})$ and the abdominal hair was removed using a depilatory cream (Veet, London, UK); the animals were divided into four groups with six animals in each group. After allowing $24 \mathrm{~h}$ for SC recovery, a container was fixed onto the abdominal area with superglue to administer liquid sample without leakage, and 0.5 g FK506-NIC complex solution, FK506-NIC-CS-NPs, FK506 suspension, and commercial preparation of FK506 (Protopic) were added into the container, respectively. After $24 \mathrm{~h}$ of in vivo permeation, the permeated skin was carefully cleaned and tape stripping was done twice with an adhesive tape (Scotch ${ }^{\circledR}$ Transparent Tape, 3M Corporate, St Paul, MN, USA) to remove any residual formulations from the skin. Subsequently, tape stripping was continuously carried out 20 times to strip the SC, with each tape stripping at a pressure of $200 \mathrm{~g}$ and the tape sticking to the skin for $10 \mathrm{~s}$. The stripped SC was collected in a centrifuge tube. Afterward, the viable epidermis and dermis were isolated from Sprague Dawley rats, cut into small pieces, and transferred into another centrifuge tube. The FK506 in the SC and viable epidermis and dermis was extracted, treated, and analyzed as described in in vitro permeation.

The FK506 content of all the samples in this study was determined with a validated HPLC method. The HPLC system (Agilent 1260 series, USA) composed of a quaternary pump (G1310A), degasser (G1322A), auto-sampler (G1329), column thermostat (CO-1000), UV detector (G1314A), and data-processing software (Agilent Chem Station for LC systems). FK506 was separated through a C8 column (Grace ${ }^{\circledR}, 250 \times 4.6 \mathrm{~mm}, 5 \mu \mathrm{m}$ ) with water/isopropyl alcohol/ tetrahydrofuran $(5 / 2 / 2, v / v / v)$ as the mobile phase at a flow rate of $0.8 \mathrm{~mL} / \mathrm{min}$ at $55^{\circ} \mathrm{C}$, and the detection wavelength was set at $220 \mathrm{~nm}$.

\section{Animal studies}

\section{$A D$ model establishment and treatment}

To induce AD-like skin lesions, DNCB was topically applied to $\mathrm{BABL} / \mathrm{c}$ mice. ${ }^{49}$ Briefly, hair on the dorsal skin of the mice was removed by shaving and recovered for $24 \mathrm{~h}$. 
DNCB was solubilized in acetone/olive oil/alcohol (2/1/1, $\mathrm{v} / \mathrm{v} / \mathrm{v})$ to prepare $0.5 \%$ and $1.0 \%(\mathrm{w} / \mathrm{v})$ DNCB solution, and $0.5 \%(\mathrm{w} / \mathrm{v})$ DNCB solution was used to sensitize the mice through once-daily application of $200 \mu \mathrm{L}$ onto the shaved area and $20 \mu \mathrm{L}$ onto the right ear for three consecutive days. Thereafter, the sensitized skin was challenged with $100 \mu \mathrm{L}$ of $1.0 \%(\mathrm{w} / \mathrm{v}) \mathrm{DNCB}$ solution on the back and $10 \mu \mathrm{L}$ on the right ear on days $4,7,11,14,17,20,23,26$, and 28 , respectively.

Mice were randomly divided into eight groups ( $n=6$ per group) as follows: 1) control: healthy mice with shaved dorsal region; 2) DNCB: AD-induced mice without treatment; 3) DNCB+Protopic: AD-induced mice treated with Protopic; 4) DNCB+Vehicle: AD-induced mice treated with NIC-CS-NPs vehicle containing 20\% (w/v) NIC; 5) $\mathrm{DNCB}+\mathrm{C}-0.01$ : AD-induced mice treated with FK506NIC-CS-NPs containing 20\% (w/v) NIC and 0.01\% (w/v) FK506; 6) DNCB+C-0.03: AD-induced mice treated with FK506-NIC-CS-NPs containing 20\% (w/v) NIC and 0.03\% (w/v) FK506; 7) DNCB+C-0.05: AD-induced mice treated with FK506-NIC-CS-NPs containing 20\% (w/v) NIC and $0.05 \%$ (w/v) FK506; and 8) DNCB+C-0.1: AD-induced mice treated with FK506-NIC-CS-NPs containing 20\% (w/v) NIC and $0.1 \%(w / v)$ FK506. Except for the control group, each mouse of the other seven groups was sensitized and challenged with DNCB as mentioned earlier. The DNCB group, the disease model group, was only sensitized and challenged with DNCB without any treatment with other formulations. For the treated groups 3-8, the sensitized mouse was topically treated with different formulations of $200 \mu \mathrm{L}(\mu \mathrm{g})$ on the shaved area and $20 \mu \mathrm{L}(\mu \mathrm{g})$ on the right ear, respectively, following each challenge since Day 4. The DNCB+Vehicle group was treated with NIC-CS-NPs vehicle without FK506, which was to confirm whether the vehicle containing NIC ameliorated AD. Different formulations containing FK506, including Protopic, $\mathrm{C}-0.01, \mathrm{C}-0.03, \mathrm{C}-0.05$, or $\mathrm{C}-0.1$, were topically administered, respectively, onto the shaved area and right ear for comparing their therapeutic effects.

\section{Evaluation of dermatitis severity}

Throughout the AD model construction and treatment, clinical dermatitis severity was visualized and scored on days 4 , 7,14 , and 28. Clinical symptoms of AD including erythema/ hemorrhage, scarring/dryness, edema, and excoriation/ erosion were scored as follows: 0) (none), 1) (mild, <20\%), 2) (moderate, 20\%-60\%), and 3) (severe, $>60 \%$ ). ${ }^{50,51}$ The sum of the four individual scores was defined as the dermatitis severity score.
On the day after the last model construction and treatment (Day 29), the thickness of the treated ear of each mouse was measured with a digimatic micrometer (CD-6 CSX, Mitutoyo Corporation, Japan), the body weight was measured, and blood samples were collected. After blood collection, the mice were sacrificed to obtain the dorsal skin and spleen. The spleen was weighed and the spleen index was calculated with spleen weight to the body weight. The dorsal skin was subjected to histopathological analysis.

\section{Histopathological studies}

The dorsal skin was fixed in $10 \%(\mathrm{w} / \mathrm{v})$ paraformaldehyde in $0.1 \mathrm{M}$ phosphate-buffered solution (PBS; pH 7.4), embedded in paraffin, sliced into 3-5 $\mu \mathrm{m}$ sections, and stained with hematoxylin and eosin (HE) and toluidine blue (TB) in accordance with standard procedures to monitor histological changes in the skin and recruitment of mast cells, respectively. To assess inflammatory cells and mast cells, slides were observed with light microscopy at 100× magnification, and cells were counted in a high-power field (HPF) at 200× magnification.

\section{Serum total IgE measurement}

Serum samples were obtained from the collected blood through centrifugation at 2,000 rpm for $30 \mathrm{~min}$, and were stored at $-80^{\circ} \mathrm{C}$ until use. Levels of total $\mathrm{IgE}$ in the serum were measured using a mouse enzyme-linked immunosorbent assay (ELISA) kit (Abcam, UK) according to the instructions of the manufacturer.

\section{Statistical analysis}

All experimental data are expressed as mean \pm standard deviation and were analyzed using one-way ANOVA, followed by the least significant difference test (LSD) as post hoc analysis (SPSS version 19.0; SPSS Inc., Chicago, IL, USA); $P<0.05$ was set as indicative of statistically significant difference.

\section{Results and discussion Preparation and characterization of FK506-NIC-CS-NPs}

FK506, a poorly aqueous soluble and high-molecular-weight drug, permeates into the skin with difficulty and can be loaded into CS-NPs. Our previous report demonstrated that the hydrophilic NIC increased FK506 solubility, enhanced FK506 percutaneous permeability, and improved EE by hyaluronic acid-cholesterol (HA-Chol) NPs through FK506NIC complex formation; $20 \%$ (w/v) NIC was chosen as the 
Table I Characterization of CS-NPs and FK506-NIC-CS-NPs with $0.1 \%$ (w/v) FK506

\begin{tabular}{llllll}
\hline Sample & NIC $(\%, w / v)$ & Size $(\mathbf{n m})$ & PDI & ZP (mV) & EE (\%) \\
\hline CS-NPs & - & $187.8 \pm 1.9$ & $0.13 \pm 0.02$ & $25.3 \pm 0.1$ & - \\
FK506-NIC-CS-NPs & 5 & $109.1 \pm 6.7$ & $0.21 \pm 0.04$ & $21.8 \pm 0.3$ & $73.8 \pm 1.3$ \\
FK506-NIC-CS-NPs & 10 & $108.0 \pm 7.3$ & $0.20 \pm 0.01$ & $20.3 \pm 0.5$ & $86.3 \pm 1.1$ \\
FK506-NIC-CS-NPs & 20 & $110.2 \pm 7.9$ & $0.20 \pm 0.02$ & $19.7 \pm 0.8$ & $92.2 \pm 2.2$ \\
\hline
\end{tabular}

Notes: Data represents mean $\pm \mathrm{SD}, \mathrm{n}=3$ /group.

Abbreviations: CS-NPs, chitosan nanoparticles; FK506, tacrolimus; NIC, nicotinamide; FK506-NIC-CS-NPs, tacrolimus-loaded chitosan nanoparticles containing nicotinamide; PDI, polydispersity index; ZP, zeta potential; EE, encapsulation efficiency.

optimal hydrotrope in the system of HA-Chol-NPs. ${ }^{21}$ In this study, the FK506-NIC complex was prepared and loaded into CS-NPs with 20\% (w/v) NIC.

The CS-NPs were obtained by ionotropic gelation upon the addition of TPP to the CS solution. In our preliminary experiments, molecular weight of CS, concentration of CS, TPP concentration, and the ratio of CS/TPP were screened on the basis of the formation of CS-NPs. CS with a viscosity of 5-20 $\mathrm{mPa} \cdot \mathrm{s}$ measured in $0.5 \%$ acetic acid at $20^{\circ} \mathrm{C}$ (CS $0.5 \%$, $\mathrm{w} / \mathrm{v}$ ) was chosen, and CS solution was prepared to a concentration of $2.0 \mathrm{mg} / \mathrm{mL}$, with a $\mathrm{pH}$ value of 5.0 adjusted by $2 \mathrm{M}$ sodium hydroxide solution. The TPP solution was prepared at a concentration of $8.0 \mathrm{mg} / \mathrm{mL}$. As shown in Table 1, at the ratio of CS/TPP of 5/1, the particle size, PDI, and ZP of CS-NPs were $187.8 \pm 1.9 \mathrm{~nm}, 0.13 \pm 0.02$, and $25.3 \pm 0.1 \mathrm{mV}$, respectively. After being loaded with the FK506-NIC complex with different NIC concentrations of $5 \%, 10 \%$, and $20 \%(\mathrm{w} / \mathrm{v})$, the particle size decreased to $\sim 110 \mathrm{~nm}$, which was a suitable size for treatment of inflamed skin. ${ }^{52}$ The ZP slightly decreased to $\sim 20 \mathrm{mV}$. For FK506 encapsulation, free FK506 was hardly encapsulated in CS-NPs due to its high hydrophobicity based on our preliminary experiment. However, the EE increased from $73.8 \% \pm 1.3 \%$ to $92.2 \% \pm 2.2 \%$ with increase in the NIC concentration of $5 \%-20 \%(\mathrm{w} / \mathrm{v})$. These results indicate that NIC can significantly decrease the particle size of NPs and facilitate the encapsulation of FK506 by CS-NPs, and 20\% (w/v) NIC in the FK506-NICCS-NPs suspension was superior to the other concentration levels. The increased solubility and decreased lipophilicity of FK506 by the FK506-NIC complex are ascribed to the increased EE.

The $\mathrm{pH}$ value influences the formation and stability of CS-NPs. Therefore, FK506-NIC-CS-NPs were prepared by CS solution with $\mathrm{pH}$ values of 4.5, 5.0, and 5.5, respectively. Upon addition of NIC, the eventual $\mathrm{pH}$ of CS-NPs was 5.0, 5.4, and 5.9, respectively. To investigate the stability of CS-NPs at different $\mathrm{pH}$ values, FK506-NIC-CS-NPs were maintained at $4^{\circ} \mathrm{C}$ for 10 days. On days $0,1,3,5$, and 10 , the size and PDI of these NPs were measured by DLS. Figure 1A and $\mathrm{B}$ present the variation of size and PDI with the time at different $\mathrm{pH}$ values. The size of FK506-NIC-CS-NPs was stable throughout 10 days at $\mathrm{pH} 5.0$ and 5.4, whereas it
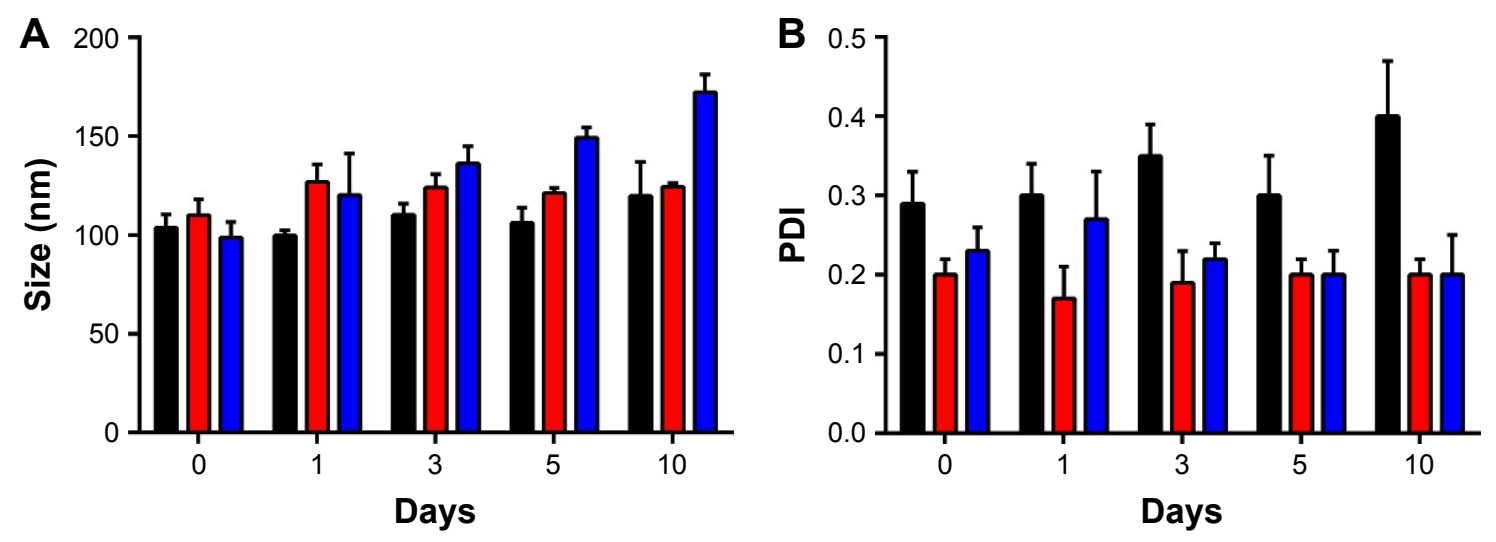

$\mathrm{pH}=5.0$

$\mathrm{pH}=5.4$

$\mathrm{pH}=5.9$

Figure I Stability of FK506-NIC-CS-NPs with 0.1\% (w/v) FK506 at different pH values in size (A) and PDI (B).

Note: Each symbol and bar represented the mean \pm SD of three determinations.

Abbreviations: FK506, tacrolimus; FK506-NIC-CS-NPs, tacrolimus-loaded chitosan nanoparticles containing nicotinamide; PDI, polydispersity index; SD, standard deviation. 

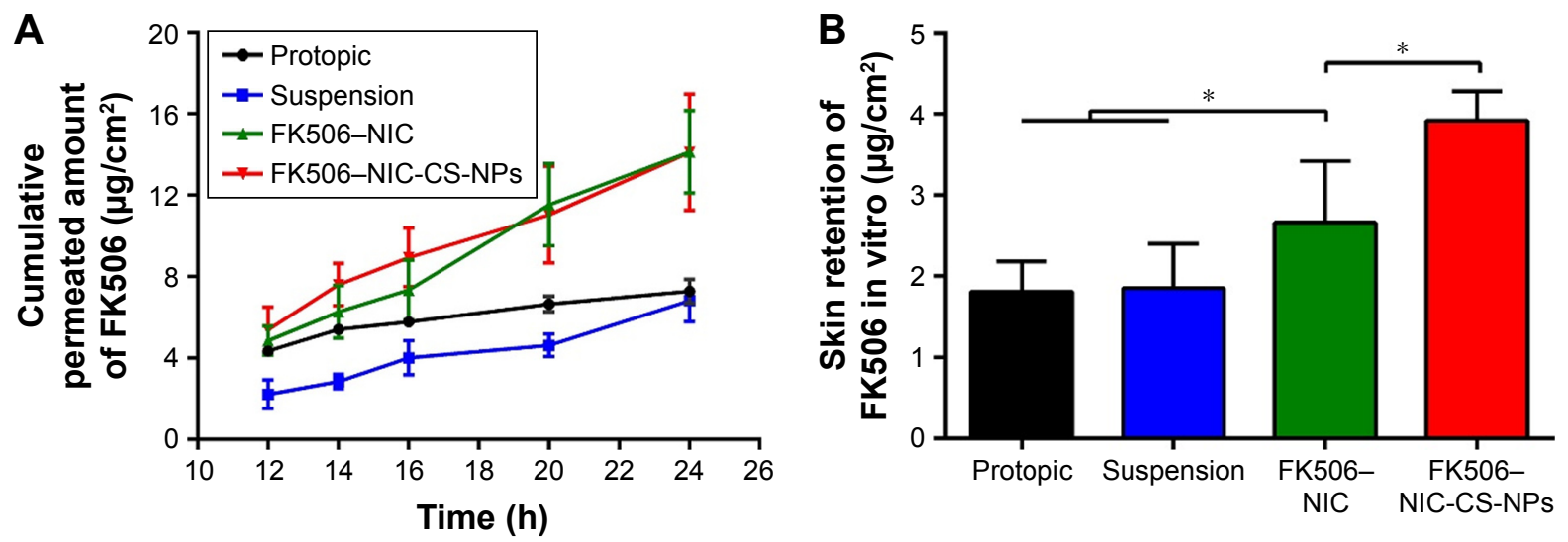

Figure 2 Permeation profiles of FK506 through rat skin from different formulations in vitro (A) and FK506 skin retention after 24-h permeation of different formulations in vitro (B).

Notes: Each symbol and bar represented the mean \pm SD of six determinations. Significant differences were calculated using ANOVA test ( $* P<0.05)$. The concentration of FK506 in each formulation was $1 \mathrm{mg} / \mathrm{mL}(\mathrm{g})$.

Abbreviations: FK506, tacrolimus; FK506-NIC, FK506 dissolved in 20\% nicotinamide aqueous solution; FK506-NIC-CS-NPs, tacrolimus-loaded chitosan nanoparticles containing nicotinamide; h, hours; SD, standard deviation.

significantly increased at $\mathrm{pH}$ 5.9. The increase of NPs size might be due to the solubility of $\mathrm{CS}$ decreasing at $\mathrm{pH}>5.5$ and to the tendency of CS to aggregate. The PDI of NPs at the resultant $\mathrm{pH} 5.0$ after 10 days was $0.40 \pm 0.07$, indicating that the distribution of particle size became scattered, whereas the PDI of NPs at pH 5.4 was still stable and remained $\sim 0.20$. Taken together, FK506-NIC-CS-NPs were prepared with $\mathrm{CS}$ solution with $\mathrm{pH}$ value of 5.0, and the resultant $\mathrm{pH}$ value turned to 5.4 after loading of the FK506-NIC complex.

\section{Skin permeation of FK506-NIC-CS-NPs in vitro}

Figure 2A and B present the profile of the FK506 cumulative permeated amount to time and the retention of FK506 in the skin after 24-h permeation, respectively. The slope of the profile of FK506 cumulative permeated amount to time is taken as the flux of FK506 from different formulations (Table 2). The commercial preparation of Protopic $(0.1 \%$, $\mathrm{w} / \mathrm{v}$ ) as a reference group and $0.1 \%(\mathrm{w} / \mathrm{v})$ FK506 aqueous suspension as an aqueous control group presented similar behaviors of permeation and retention. However, the FK506 permeation from the FK506-NIC complex or FK506-NICCS-NPs was significantly higher than that from the Protopic or FK506 suspension throughout 24-h permeation $(P<0.05)$; the cumulative permeated amount from the FK506-NIC complex and FK506-NIC-CS-NPs after 24-h permeation was $14.12 \pm 2.02$ and $14.10 \pm 2.86 \mu \mathrm{g} \mathrm{cm}^{-2}$, respectively; this was $\sim 2$-fold that of the Protopic or FK506 suspension, and there was no significant difference between the FK506-NIC complex and FK506-NIC-CS-NPs $(P>0.05)$. The flux of FK506 from different formulations was FK506-NIC > FK506-NIC-CS-NPs $>$ Protopic $\approx$ suspension. After $24-\mathrm{h}$ permeation, the skin retention of FK506 from the FK506NIC complex or FK506-NIC-CS-NPs was significantly higher than that of Protopic or FK506 suspension $(P<0.05)$. Interestingly, FK506-NIC-CS-NPs presented the highest FK506 retention in the skin, significantly higher than that of FK506-NIC $\left(P<0.05,3.92 \pm 0.36 \mu \mathrm{g} \mathrm{cm}^{-2}\right.$ vs $2.66 \pm 0.76$ $\mu \mathrm{g} \mathrm{cm}^{-2}$ ), although there was no significant difference of cumulative permeated amount between the FK506-NIC

Table 2 Cumulative permeated amount, retention, and flux of FK506 from different formulations after 24-h permeation in vitro

\begin{tabular}{llll}
\hline & $\begin{array}{l}\text { Permeated amount } \\
\left(\mu \mathbf{g ~ c m}^{-2}\right)\end{array}$ & $\begin{array}{l}\text { Retention } \\
\left(\mu \mathbf{g ~ c m}^{-2}\right)\end{array}$ & $\begin{array}{l}\text { Flux } \\
\left(\mu \mathbf{~ c m}^{-2} \mathbf{h}^{-1}\right)\end{array}$ \\
\hline Protopic & $7.28 \pm 0.58$ & $1.81 \pm 0.37$ & 0.23 \\
Suspension & $6.81 \pm 1.05$ & $1.85 \pm 0.55$ & 0.21 \\
FK506-NIC & $14.12 \pm 2.02^{* * * *}$ & $2.66 \pm 0.76^{* * * *}$ & 0.79 \\
FK506-NIC-CS-NPs & $14.10 \pm 2.86^{* * * *}$ & $3.92 \pm 0.36^{* * * * * * * *}$ & 0.69 \\
\hline
\end{tabular}

Notes: $* P<0.05$ in comparison with Protopic, $* * P<0.05$ in comparison with FK506 aqueous suspension, and $* * * P<0.05$ in comparison with FK506-NIC. Data represents mean $\pm S D, n=6 /$ group.

Abbreviations: FK506, tacrolimus; FK506-NIC, FK506 dissolved in 20\% nicotinamide aqueous solution; FK506-NIC-CS-NPs, tacrolimus-loaded chitosan nanoparticles containing nicotinamide. 
complex and FK506-NIC-CS-NPs. It can be concluded from the in vitro permeation that NIC hydrotropy can enhance the permeation of FK506 through and into the skin, and combination with CS-NPs can synergistically enhance the retention of FK506 in the skin without affecting the penetration of FK506.

\section{Skin permeation of FK506-NIC-CS-NPs in vivo}

In vivo skin permeation studies were conducted to understand the drug distribution in different skin layers and further confirm the enhancing effects on drug retention in the skin. As shown in Figure 3, after 24-h vivo permeation, FK506 permeated into the skin and was mainly retained in the SC. The FK506 retention in the SC of NIC-CS-NPs with $5.72 \pm 0.92 \mu \mathrm{g} \mathrm{cm}^{-2}$ was significantly higher than that of the NIC complex with $4.90 \pm 1.20 \mu \mathrm{g} \mathrm{cm}^{-2}(P<0.05)$, and either of them was further significantly higher than that of Protopic with $2.81 \pm 0.32 \mu \mathrm{g} \mathrm{cm}^{-2}$ or suspension with $3.37 \pm 0.57 \mu \mathrm{g} \mathrm{cm}^{-2}$ $(P<0.05)$. In deeper layers of epidermis and dermis $(\mathrm{E}+\mathrm{D})$, FK506 retention of NIC-CS-NPs with $1.03 \pm 0.13 \mu \mathrm{g} \mathrm{cm}^{-2}$ or of the NIC complex with $0.96 \pm 0.20 \mu \mathrm{g} \mathrm{cm}^{-2}$ was significantly higher than that of Protopic or $0.1 \%$ FK506 suspension $(P<0.05)$; however, there was no statistical difference between NIC-CS-NPs and the NIC complex $(P>0.05)$. It can be concluded that NIC-CS-NPs exert enhancing effects on FK506 skin permeability in vivo and deposit the drug in both

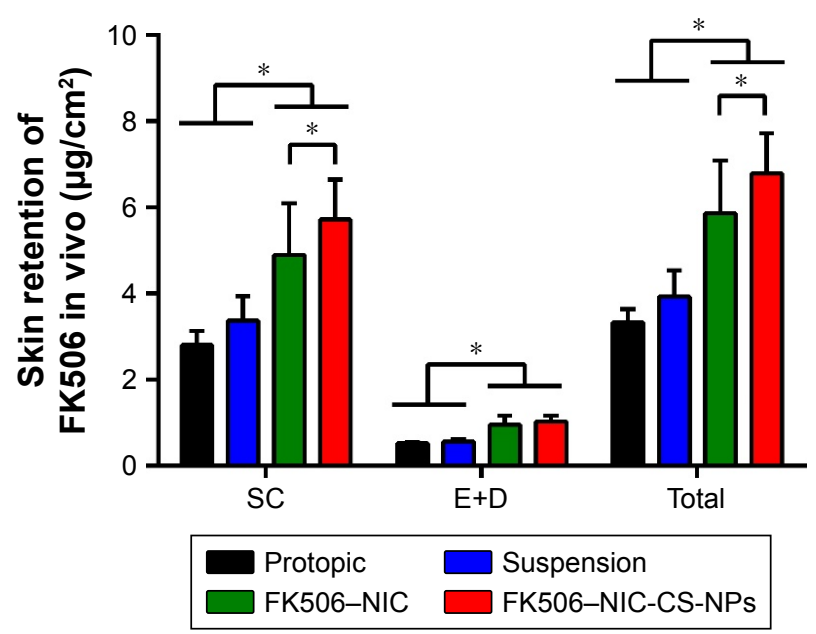

Figure 3 In vivo skin retention of FK506 from different formulations in different skin layers of SC, E+D, and total amount in skin (Total).

Notes: Each symbol and bar represented the mean \pm SD of six determinations. Significant differences were calculated using ANOVA test $(* P<0.05)$. The concentration of FK506 in each formulation was $1 \mathrm{mg} / \mathrm{mL}(\mathrm{g})$.

Abbreviations: E+D, epidermis and dermis; FK506, tacrolimus; FK506-NIC, FK506 dissolved in $20 \%$ nicotinamide aqueous solution; FK506-NIC-CS-NPs, tacrolimusloaded chitosan nanoparticles containing nicotinamide; h, hours; SC, stratum corneum; SD, standard deviation. the upper and deeper layers of the skin. Based on the total FK506 retention from the SC, epidermis, and dermis, FK506 retention in the skin from different formulations after 24-h permeation was consistent with the results from in vitro permeation, which was FK506-NIC-CS-NPs > FK506-NIC > suspension $\approx$ Protopic. An in vivo permeation study confirms that NIC hydrotropy can enhance FK506 skin permeability, and combination with CS-NPs can synergistically enhance the retention of FK506 in the skin. Furthermore, the results further demonstrate that the enhancing effect of CS-NPs on FK506 skin permeability was predominantly with regard to retention in the SC. The enhancing amount of FK506 in the $\mathrm{SC}$ and the deeper layers of epidermis and dermis by NICCS-NPs will lead to enhanced efficacy for treating AD.

Taken together, the in vitro and in vivo permeation, enhanced skin permeability, and retention of FK506 by NIC-CS-NPs ascribed to the effects of NIC and NPs as follows: 1) As reported in our previous study, ${ }^{21}$ the increased solubility of FK506 by NIC hydrotropy increased the FK506 concentration gradient and partition in skin/vehicle. Further, the FK506-NIC complex formed or self-aggregated NIC segregated FK506 from water in the NIC hydrotropic system, causing more skin permeation of FK506 under the driving force from NIC permeation. Therefore, NIC exerts enhancing FK506 skin permeability in both systems of the FK506-NIC complex and FK506-NIC-CS-NPs. 2) NPs with diameter more than $10 \mathrm{~nm}$ are unlikely to penetrate through the SC into viable human skin but tend to accumulate in hair follicle openings and furrows, resulting in the formation of a drug depot on the skin surface for sustained release and permeation in the skin without damaging the natural barrier of the skin. ${ }^{31,53-56}$ Moreover, CS works as a permeation enhancer due to its positive charges and mucoadhesive properties. Consequently, the FK506-NIC-CS-NPs system exploited both virtues of NIC and NPs to obtain synergetic effects on FK506 percutaneous delivery. Inflammatory cutaneous disorders such as AD might be treated more effectively with the enhanced percutaneous FK506 delivery to the specific site of the skin by NIC-CS-NPs, which also provides the possibility of reducing the dose of FK506. However, that would be the subject of subsequent research.

\section{Effects of FK506-NIC-CS-NPs on ameliorating AD-like clinical symptoms}

The in vitro or in vivo skin-permeation studies mentioned earlier have shown that greater amounts of FK506 could be obtained in the skin through enhanced percutaneous FK506 delivery by NIC-CS-NPs, and the dose of FK506 may be 
reduced owing to its enhanced delivery. In addition, NICcontaining moisturizers are known to be effective in alleviating dry skin conditions, reducing transepidermal water loss (TEWL), enhancing biosynthesis of ceramide, and strengthening the skin barrier for treatment of acne, rosacea, $\mathrm{AD}$, and even skin cancer. ${ }^{57-60} \mathrm{NIC}$ is a promising topical formulation adjuvant with unique $\mathrm{SC}$ barrier-augmentation properties. ${ }^{61}$ Therefore, NIC may play dual roles in the FK506-NIC-CSNPs system of enhancing FK506 percutaneous delivery and alleviating skin disorders of $\mathrm{AD}$.

In the present study, we compared the effects of FK506-NIC-CS-NPs containing different doses of FK506 on ameliorating DNCB-induced AD-like clinical symptoms. Figure 4A describes the schematic diagram of the experimental protocol in the AD model mouse. Figure 4B presents the dermatitis severity scores of $\mathrm{AD}$ model mice treated with different formulations along the time graph, and Figure 4C showed the clinical features of mice after 28-day treatment with different formulations. After three consecutive days of sensitization with $0.5 \% \mathrm{DNCB}$, the severity of dryness in the skin increased in comparison with the control group. After challenge, mouse skin displayed typical characteristics of AD clinical symptoms including erythema/hemorrhage, scarring/dryness, edema, and excoriation/erosion, and the symptoms progressively intensified for the DNCB group. After treatment with different formulations, these four symptoms were alleviated at different levels in comparison with the DNCB group. As shown in Figure 4B, the sum score of the four symptoms of each group decreased throughout 28 days of treatment with different formulations. However, the dermatitis score of the $\mathrm{DNCB}+$ Protopic group significantly decreased until Day $28(P<0.05)$; there was no significant difference between the $\mathrm{DNCB}+$ Protopic and DNCB groups prior to Day $21(P>0.05)$. It has been reported that the ointment vehicle of Protopic induced skin irritation because of its undesirable stickiness, greasiness, and not water washability, resulting in an uneasy feeling in patients, and had a limitation in releasing drug into the skin. ${ }^{27}$ These issues related to Protopic might ascribe to the higher dermatitis scores in the first 3 weeks. Moreover, the significant decrease of dermatitis scores occurring after 28-day treatment with Protopic demonstrated that long-term treatment with Protopic is necessary for alleviating AD-like symptoms. Interestingly, as we expected, NIC-CS-NPs vehicle without FK506 loading presented similar ameliorating effects on the four symptoms of $\mathrm{AD}$ as with Protopic after 28-day treatment,
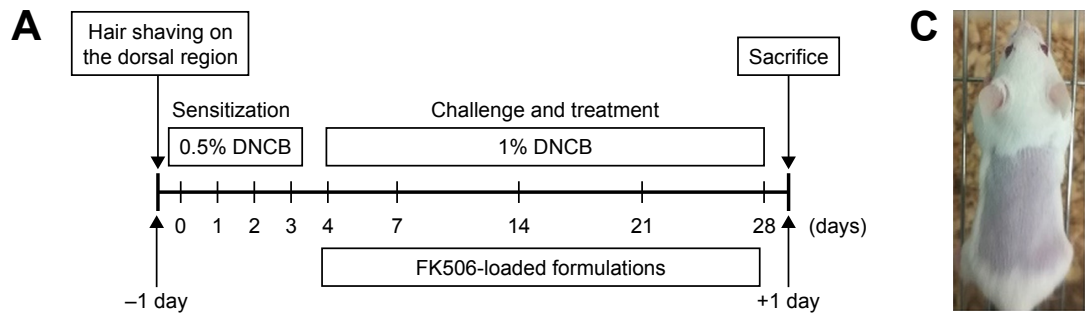

B

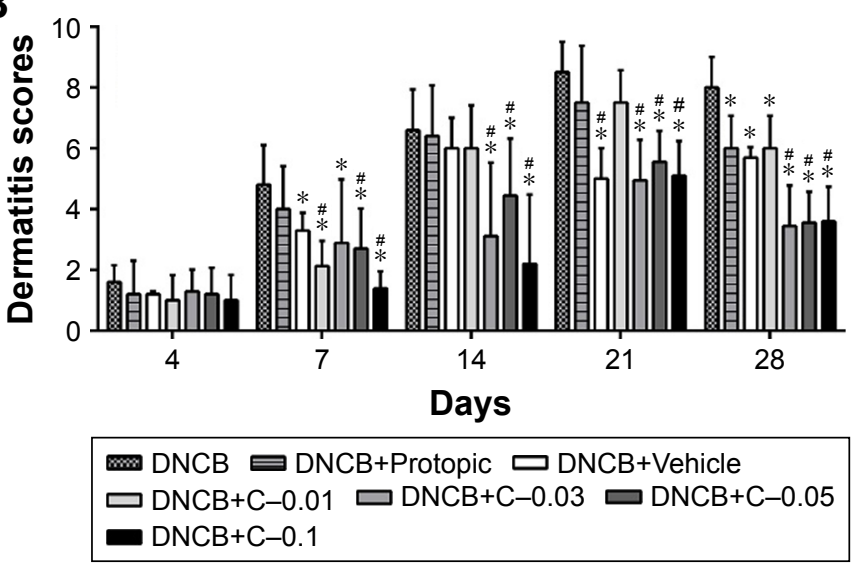

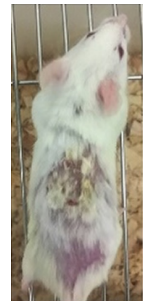

DNCB

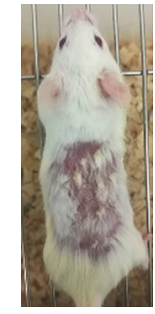

DNCB+

C-0.01

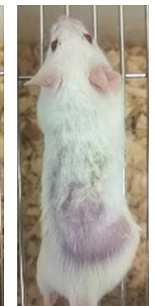

DNCB+

C-0.03

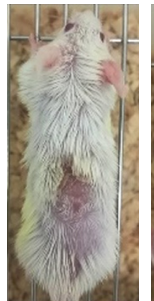

DNCB+ Protopic

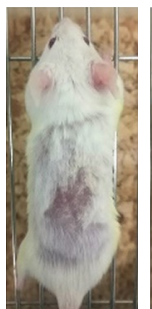

DNCB+

C-0.05

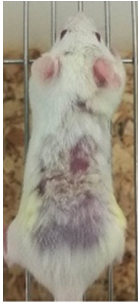

DNCB+

Vehicle

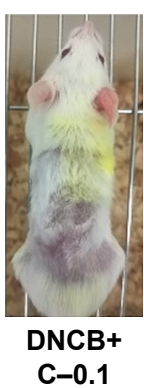

Figure 4 Schematic diagram of the experimental protocol in a mouse model (A). Dermatitis scores of AD-model mice treated with different formulations (B) and representative clinical features of $A D$-like skin lesions at the end of the experiment (C).

Notes: Each bar represented the mean \pm SD of six determinations. Significant differences were calculated using ANOVA test. $* P<0.05$ in comparison with the DNCB group; ${ }^{\#} P<0.05$ in comparison with the DNCB+Protopic group.

Abbreviations: AD, atopic dermatitis; C-0.0I, FK506-NIC-CS-NPs containing 20\% (w/v) NIC and 0.01\% (w/v) FK506; C-0.03, FK506-NIC-CS-NPs containing 20\% (w/v) NIC and $0.03 \%$ (w/v) FK506; C-0.05, FK506-NIC-CS-NPs containing 20\% (w/v) NIC and 0.05\% (w/v) FK506; C-0.I, FK506-NIC-CS-NPs containing 20\% (w/v) NIC and 0.I\% (w/v) FK506; DNCB, I-chloro-2,4-dinitrobenzene; FK506, tacrolimus; FK506-NIC-CS-NPs, tacrolimus-loaded chitosan nanoparticles containing nicotinamide; NIC, nicotinamide. 
which were ascribed to the positive effects of NIC on AD skin. NIC was shown to have beneficial effects on various cutaneous inflammatory disorders including AD; it induces antipruritic effects through recovering the skin barrier, and anti-inflammation by inhibiting PARP-1 and the associated expression of inflammatory cytokines, chemokines, adhesion molecules, and inflammatory mediators. ${ }^{62,63}$ NIC might be worthwhile in basic therapy of AD. Based on literature reports and our study, NIC was expected to play an adjuvant role in the FK506-NIC-CS-NPs system for AD treatment, and consequently reduce the dose of FK506. Therefore, the effects of FK506-NIC-CS-NPs containing different doses of FK506 (0.01\%, 0.03\%, 0.05\%, and 0.1\%, w/v) on ameliorating DNCB-induced AD-like skin lesions were further compared with Protopic $(0.1 \%, w / w)$. As shown in Figure 4B, the dermatitis score of FK506-NIC-CS-NPs with FK506 of $0.03 \%, 0.05 \%$, or $0.1 \%(\mathrm{w} / \mathrm{v})$ was significantly lower than that of the DNCB group since Day $7(P<0.05)$, and the score of FK 506-NIC-CS-NPs with FK506 of $0.05 \%$ or $0.1 \%$ (w/v) was further significantly lower than that of the Protopic group since Day $7(P<0.05)$. After day 14, the score of NPs with FK506 of $0.03 \%, 0.05 \%$, or $0.1 \%(\mathrm{w} / \mathrm{v})$ was significantly lower than that of Protopic throughout the treatment period $(P<0.05)$. However, the score of NPs with FK 506 concentration of $0.01 \%(\mathrm{w} / \mathrm{v})$ presented a similar trend to that of the Protopic group $(P>0.05)$. Figure 4C shows the treatment of NIC-CS-NPs containing 0.1\% (w/v) FK506 or lower doses of $0.05 \%$ and $0.03 \%(\mathrm{w} / \mathrm{v})$ that almost completely inhibited
DNCB-induced external changes of the skin. These results indicated that, with the help of NIC-CS-NPs, $0.1 \%(\mathrm{w} / \mathrm{v})$ FK506 or lower doses of $0.05 \%$ and $0.03 \%(\mathrm{w} / \mathrm{v})$ had higher efficacy in ameliorating the development of AD-like skin symptoms than $0.1 \%$ (w/w) FK506 ointment, and even NIC-CS-NPs with $0.01 \%$ FK506 (w/v) or without FK506 presented comparable efficacy in ameliorating the development of AD-like skin symptoms to Protopic. In conclusion, compared to Protopic, the NIC-CS-NPs drug-delivery system can decrease the dose of FK506 in ameliorating the development of AD-like skin symptoms.

The ear swelling and splenic weight increase revealed the inflammation mediated by DNCB. The ear thickness was measured to evaluate ear swelling, and the weight of the spleen was measured to calculate the spleen index (spleen index, $\%=$ weight of spleen/weight of body $\times 100 \%)$. As shown in Figure 5A, ear thickness significantly increased in the DNCB group; however, treatment with different formulations significantly inhibited ear thickening, and NIC-CS-NPs with FK506 of $0.1 \%, 0.05 \%$, or $0.03 \%$ (w/v) further significantly reduced the ear thickness in comparison with Protopic. Figure 5B shows that the spleen index of the DNCB group was significantly elevated after AD-induction as compared to the control group, indicating the immune activation in the AD mouse model. However, treatment with different formulations significantly suppressed activated immunity and decreased the elevated spleen index, and NIC-CS-NPs with FK506 of $0.1 \%, 0.05 \%$, or $0.03 \%(\mathrm{w} / \mathrm{v})$ further reduced
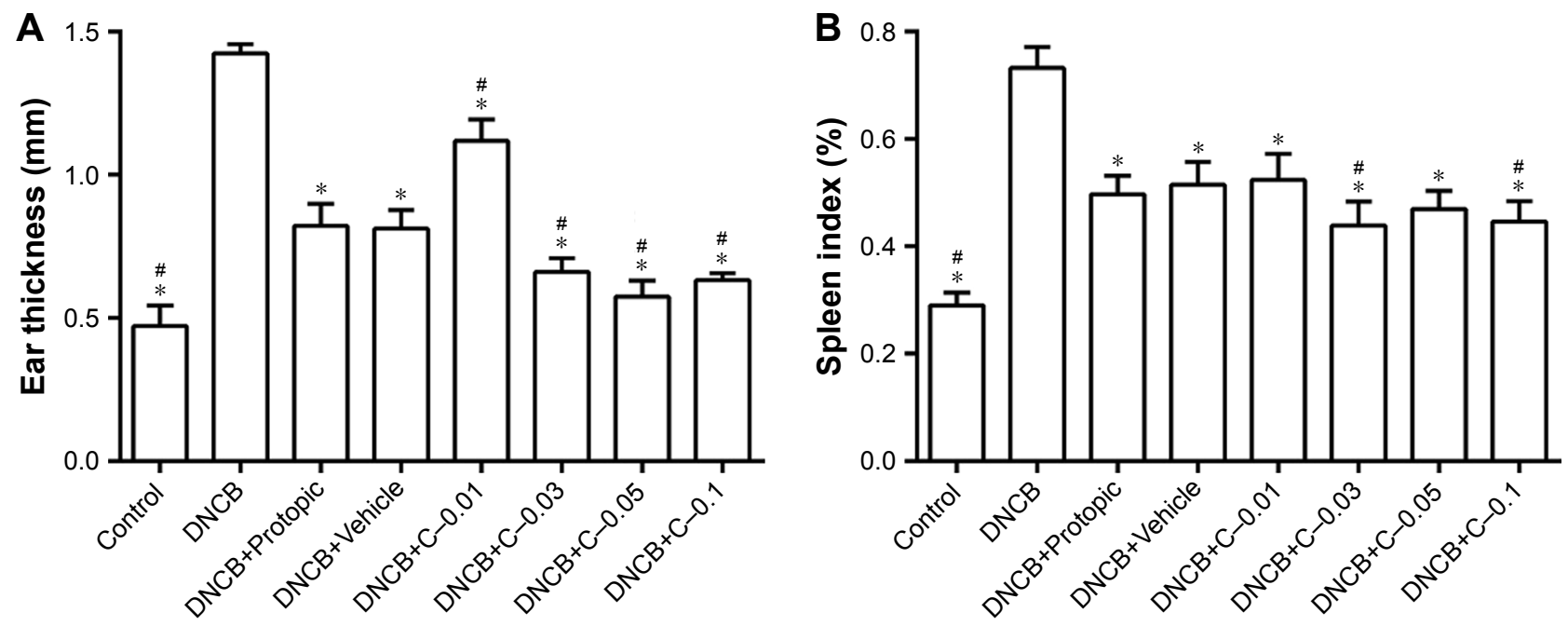

Figure 5 The ear thickness $(\mathbf{A})$ and spleen index $(\mathbf{B})$ on day 29 of mice treated with different formulations.

Notes: Each bar represented the mean \pm SD of six determinations. Significant differences were calculated using ANOVA test. $* P<0.05$ in comparison with $D N C B$ group; ${ }^{\#} P<0.05$ in comparison with the DNCB+Protopic group.

Abbreviations: C-0.0I, FK506-NIC-CS-NPs containing 20\% (w/v) NIC and 0.01\% (w/v) FK506; C-0.03, FK506-NIC-CS-NPs containing 20\% (w/v) NIC and 0.03\% (w/v) FK506; C-0.05, FK506-NIC-CS-NPs containing 20\% (w/v) NIC and 0.05\% (w/v) FK506; C-0.1, FK506-NIC-CS-NPs containing 20\% (w/v) NIC and 0.I\% (w/v) FK506; DNCB, I-chloro-2,4-dinitrobenzene; FK506, tacrolimus; FK506-NIC-CS-NPs, tacrolimus-loaded chitosan nanoparticles containing nicotinamide; NIC, nicotinamide. 
the spleen index in comparison with Protopic. Therefore, the anti-inflammatory and immunosuppressive effects of NIC-CS-NPs with $0.1 \%, 0.05 \%$, or $0.03 \%$ (w/v) FK506 were significantly stronger than that of $0.1 \%$ (w/w) FK506 Protopic ointment.

\section{Histopathological analysis}

To further understand the effects of FK506-loaded formulations on DNCB-induced AD-like symptoms in mice, the skin tissues were subjected to histological analysis to observe histological changes and infiltration of inflammatory cells such as lymphocytes and mast cells. Figure 6A presents $\mathrm{HE}$ stains of skin tissues. In DNCB-induced AD skin, the epidermis was thickened due to hypertrophy and hyperkeratosis of epidermal keratinocytes, and the dermal cell population was increased in the skin lesion; cells, particularly with round-shaped nucleus (stained in purple), were greatly accumulated. After being treated with Protopic or NIC-CS-NPs vehicle, epidermal thickness was moderately diminished, whereas epidermal thickness was significantly diminished when treated with NIC-CS-NPs containing FK506 of $0.03 \%$, $0.05 \%$, or $0.1 \%(\mathrm{w} / \mathrm{v})$, respectively. Figure $6 \mathrm{~B}$ presents the TB stain of inflammatory cells. In the DNCB-induced AD skin lesion, the number of mast cells increased and infiltrated into the epidermis and dermis. After treatment with Protopic or an NIC-CS-NPs vehicle, the number of infiltrated mast cells was partially decreased, whereas DNCB-mediated mast cell accumulation was significantly decreased when treated with NIC-CS-NPs containing FK506 of 0.03\%, 0.05\%, or $0.1 \%(\mathrm{w} / \mathrm{v})$, respectively. The results of histological analysis provided further evidence that NIC-CS-NPs with lowdose FK506, compared to Protopic, ameliorated AD-like

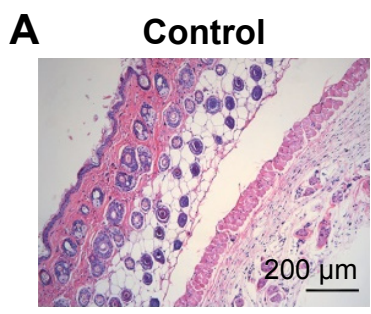

$\mathrm{DNCB}+\mathrm{C}-0.01$

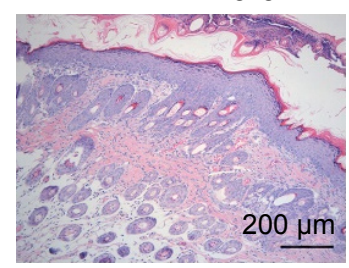

B

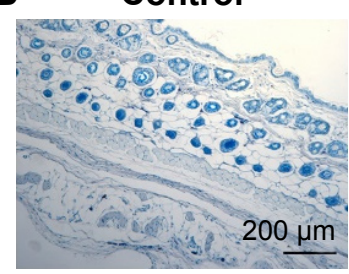

DNCB+C-0.01

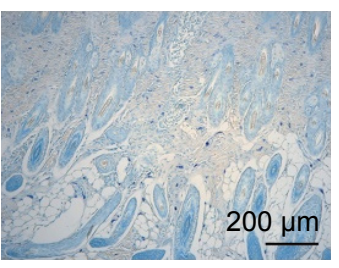

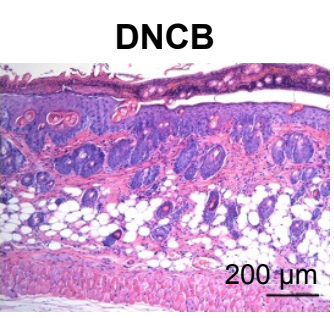

DNCB+C-0.03

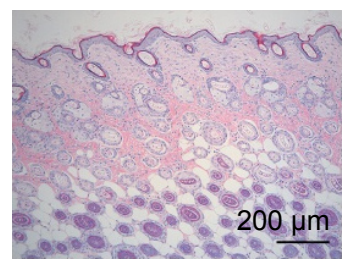

DNCB

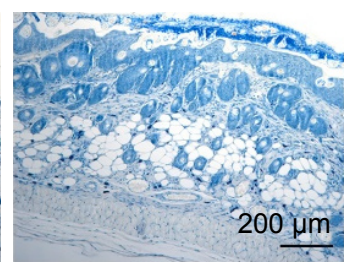

DNCB+C-0.03

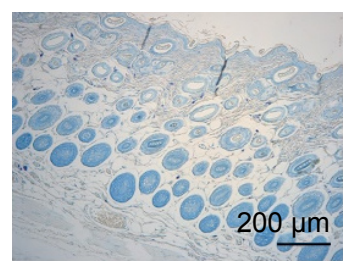

DNCB+Protopic

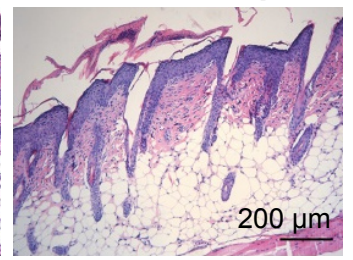

DNCB+C-0.05

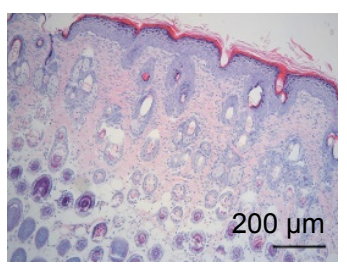

DNCB+Protopic

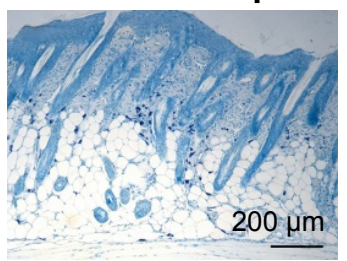

DNCB+C-0.05

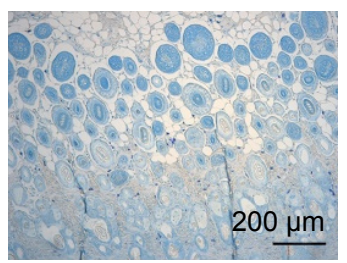

DNCB+Vehicle

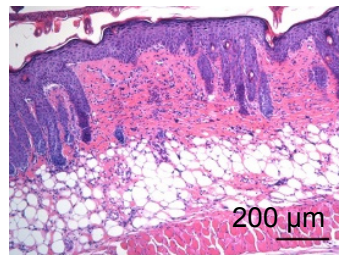

DNCB+C-0.1

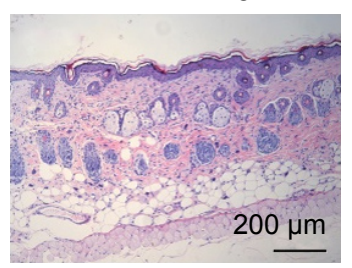

DNCB+Vehicle

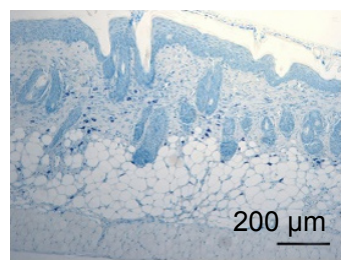

DNCB+C-0.1

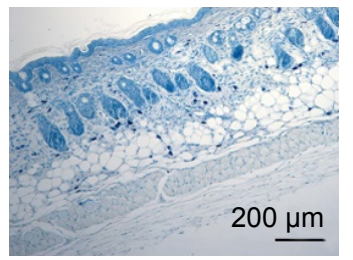

Figure 6 Histological features of skin after treatment with different formulations in the AD mouse model. The sliced sections were stained with hematoxylin and eosin (A) and toluidine blue (B) (magnification I00x). Scale bar: $200 \mu \mathrm{m}$.

Abbreviations: AD, atopic dermatitis; C-0.0I, FK506-NIC-CS-NPs containing 20\% (w/v) NIC and 0.01\% (w/v) FK506; C-0.03, FK506-NIC-CS-NPs containing 20\% (w/v) NIC and $0.03 \%$ (w/v) FK506; C-0.05, FK506-NIC-CS-NPs containing 20\% (w/v) NIC and 0.05\% (w/v) FK506; C-0.I, FK506-NIC-CS-NPs containing 20\% (w/v) NIC and 0.I\% (w/v) FK506; DNCB, I-chloro-2,4-dinitrobenzene; FK506, tacrolimus; FK506-NIC-CS-NPs, tacrolimus-loaded chitosan nanoparticles containing nicotinamide; NIC, nicotinamide. 
skin lesions remarkably after FK506 incorporation into NIC-CS-NPs.

\section{Effects of FK506-NIC-CS-NPs on downregulating serum total lgE levels}

$\mathrm{AD}$ is an IgE-mediated hypersensitive skin disease; the increase of serum total IgE levels is a typical characteristic in DNCB-induced BALB/c mice with AD-like skin lesions. ${ }^{64,65}$ As shown in Figure 7, the serum total IgE levels of DNCB group in the present study almost increased 5-fold of that in the control group. After treatment with Protopic or an NIC-CS-NPs vehicle, the serum total IgE levels was significantly downregulated to 3.5- or 2.8-fold that of the control group. Interestingly, the serum total IgE level was further significantly decreased in comparison with the Protopic group when treated with NIC-CS-NPs containing FK506 of $0.03 \%, 0.05 \%$, or $0.1 \%(\mathrm{w} / \mathrm{v})$, respectively; it was $2-, 1.6-$, or 1.8-fold of the control group, respectively. Therefore, the results confirm that NIC-CS-NPs with low-dose FK506 compared to Protopic can remarkably decrease the pathological marker protein IgE of AD after FK506 incorporation into NIC-CS-NPs.

The improved effects of NIC-CS-NPs with low-dose FK506 in comparison with Protopic in the treatment of AD-like lesions have been proved on the basis of clinical symptoms,

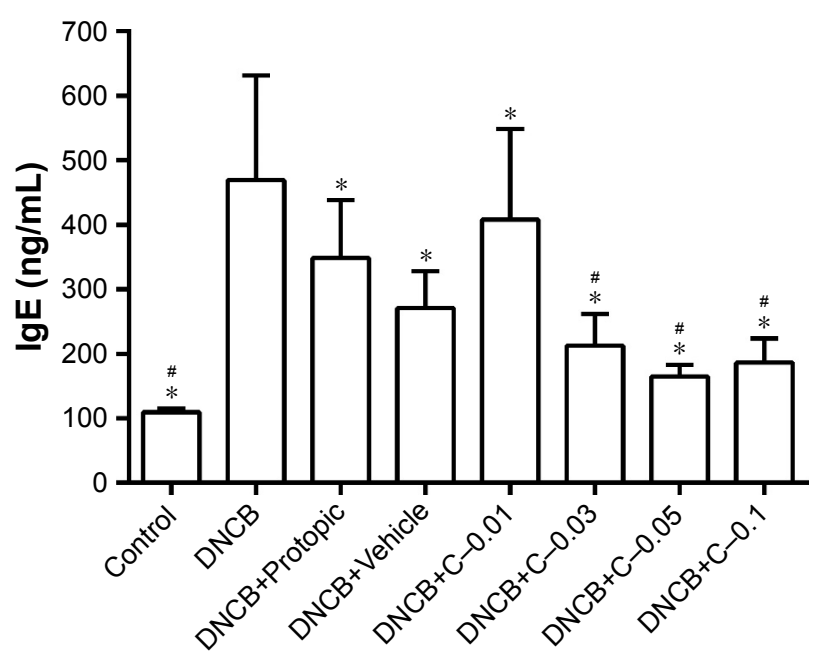

Figure 7 Effects of NPs with low-dose FK506 on serum total lgE levels.

Notes: Each bar represented the mean \pm SD of six determinations. Significant differences were calculated using ANOVA test. $* P<0.05$ in comparison with the $D N C B$ group; ${ }^{*}<<0.05$ in comparison with the $D N C B+$ Protopic group.

Abbreviations: C-0.0I, FK506-NIC-CS-NPs containing $20 \%(\mathrm{w} / \mathrm{v}) \mathrm{NIC}$ and $0.01 \%(\mathrm{w} / \mathrm{v})$ FK506; C-0.03, FK506-NIC-CS-NPs containing 20\% (w/v) NIC and $0.03 \%(w / v)$ FK506; C-0.05, FK506-NIC-CS-NPs containing $20 \%(w / v) ~ N I C$ and $0.05 \%$ (w/v) FK506; C-0.I, FK506-NIC-CS-NPs containing 20\% (w/v) NIC and $0.1 \%$ (w/v) FK506; DNCB, I-chloro-2,4-dinitrobenzene; FK506, tacrolimus; FK506-NICCS-NPs, tacrolimus-loaded chitosan nanoparticles containing nicotinamide; IgE, immunoglobulin E; NIC, nicotinamide. histological analysis, and molecular biology in our present study. The benefits of NIC-CS-NPs for FK506 on anti-AD are ascribed to the following items. First, through the synergistic enhancement of NIC and CS-NPs on FK506 percutaneous delivery, NIC-CS-NPs significantly delivered more quantities of FK506 into deeper layers of the skin under the same dose of FK506 administered. This point was proved in the in vitro and in vivo permeation studies. Consequently, NIC-CS-NPs encapsulating low-dose FK506 could reach the therapeutic dose. Second, NIC played the role of a therapeutic adjuvant in AD. Many reports have suggested that topical application of NIC had several beneficial effects, especially anti-inflammatory, antipruritic, and antimicrobial effects. ${ }^{44,66-68}$ In the present study, the vehicle group containing NIC presented comparable efficacy in ameliorating AD-like clinical symptoms, decreasing epidermis thickening and inflammatory cell infiltration, and downregulating the serum total IgE. In comparison with Protopic (containing 0.1\% FK506), the dose of FK506 through the NIC-CS-NPs system could be decreased to at least $0.03 \%$-approximately one-third the dose of Protopic. Third, CS exhibited its antibacterial and wound-healing effects in FK506-NIC-CS-NPs for anti-AD. ${ }^{69}$ Therefore, NIC-CS-NPs as a percutaneous delivery system for FK506 could reduce FK506 dose and maintain good therapeutic efficacy simultaneously.

\section{Conclusion}

Topical application of FK506 was effective in the treatment of immune-mediated cutaneous disorders; however, adverse effects frequently occurred with the increase of FK506 dose for the long-term treatment of AD. In this study, we developed an NIC-CS-NPs system containing FK506 for topical anti-AD treatment. FK506 was effectively loaded into CS-NPs with high EE and stability with the help of NIC as a hydrotropic agent. CS-NPs in combination with NIC exhibited a synergistic enhancement on FK506 permeation and retention in the skin, both in vitro and in vivo. The treatment of DNCB-induced AD-like skin lesions with FK506-NIC-CS-NPs demonstrated that NIC-CS-NPs with approximately one-third the dose of FK506 in Protopic obtained anti-AD efficacy superior to that of Protopic, and the NIC-CS-NPs vehicle exhibited moderate anti-AD effects. Overall, the results support that NIC-CS-NPs enhance the permeability of FK506, play an adjuvant role in anti-AD, and reduce the dose of FK506 in treating AD and, therefore, constitute a promising nanoscale system of FK506 for the effective treatment of AD. 


\section{Acknowledgments}

The work was financially supported by the National Natural Science Foundation of China (grant nos 81073066 and 81473358) and Science and Technology Planning Project of Guangdong Province, People's Republic of China (grant no 2017A020211020).

\section{Disclosure}

The authors report no conflicts of interest in this work.

\section{References}

1. Spergel JM, Paller AS. Atopic dermatitis and the atopic march.J Allergy Clin Immunol. 2003;112(6 Suppl):S118-S127.

2. Soter NA. Morphology of atopic eczema. Allergy. 1989;44(Suppl 9): $16-19$.

3. Bieber T. Atopic dermatitis. Ann Dermatol. 2010;22(2):125-137.

4. Abboud D, Hanson J. Chemokine neutralization as an innovative therapeutic strategy for atopic dermatitis. Drug Discov Today. 2017; 22(4):702-711.

5. Leung DY, Bieber T. Atopic dermatitis. Lancet. 2003;361(9352): 151-160.

6. Hengge UR, Ruzicka T, Schwartz RA, Cork MJ. Adverse effects of topical glucocorticosteroids. J Am Acad Dermatol. 2006;54(1):1-15; quiz 16-18.

7. Ruzicka T, Assmann T, Homey B. Tacrolimus: the drug for the turn of the millennium? Arch Dermatol. 1999;135(5):574-580.

8. Rallis E, Korfitis C, Gregoriou S, Rigopoulos D. Assigning new roles to topical tacrolimus. Expert Opin Investig Drugs. 2007;16(8):1267-1276.

9. Saeki H, Nakahara T, Tanaka A; Committee for Clinical Practice Guidelines for the Management of Atopic Dermatitis of Japanese Dermatological Association. Clinical practice guidelines for the management of atopic dermatitis 2016. J Dermatol. 2016;43(10):1117-1145.

10. Saeki H. Management of atopic dermatitis in Japan. J Nippon Med Sch. 2017;84(1):2-11.

11. El-Batawy MM, Bosseila MA, Mashaly HM, Hafez VS. Topical calcineurin inhibitors in atopic dermatitis: a systematic review and meta-analysis. J Dermatol Sci. 2009;54(2):76-87.

12. Svensson A, Chambers C, Gånemo A, Mitchell SA. A systematic review of tacrolimus ointment compared with corticosteroids in the treatment of atopic dermatitis. Curr Med Res Opin. 2011;27(7):1395-1406.

13. Noguchi A, Tominaga M, Takahashi N, et al. Difference in therapeutic effects of topically applied corticosteroid and tacrolimus on atopic dermatitis-like symptoms in NC/Nga mice. J Dermatol Sci. 2017;86(1): 54-62.

14. Danby SG, Chittock J, Brown K, Albenali LH, Cork MJ. The effect of tacrolimus compared with betamethasone valerate on the skin barrier in volunteers with quiescent atopic dermatitis. Br J Dermatol. 2014;170(4): 914-921.

15. Gupta AK, Adamiak A, Chow M. Tacrolimus: a review of its use for the management of dermatoses. J Eur Acad Dermatol Venereol. 2002;16(2): 100-114.

16. de Paulis A, Stellato C, Cirillo R, Ciccarelli A, Oriente A, Marone G. Anti-inflammatory effect of FK-506 on human skin mast cells. J Invest Dermatol. 1992;99(6):723-728.

17. Zabawski EJ, Costner M, Cohen JB, Cockerell CJ. Tacrolimus: pharmacology and therapeutic uses in dermatology. Int J Dermatol. 2000; 39(10):721-727.

18. Ruzicka T, Bieber T, Schöpf E, et al. A short-term trial of tacrolimus ointment for atopic dermatitis. European Tacrolimus Multicenter Atopic Dermatitis Study Group. N Engl J Med. 1997;337(12):816-821.

19. Soter NA, Fleischer AB Jr, Webster GF, Monroe E, Lawrence I. Tacrolimus ointment for the treatment of atopic dermatitis in adult patients: Part II, safety. J Am Acad Dermatol. 2001;44(1 Suppl):S39-S46.
20. Yamanaka M, Yokota S, Iwao Y, Noguchi S, Itai S. Development and evaluation of tacrolimus cream formulation using a binary solvent system. Int J Pharm. 2014;464(1-2):19-26.

21. Pan W, Qin M, Zhang G, et al. Combination of hydrotropic nicotinamide with nanoparticles for enhancing tacrolimus percutaneous delivery. Int J Nanomedicine. 2016;11:4037-4050.

22. Wan T, Pan J, Long Y, et al. Dual roles of TPGS based microemulsion for tacrolimus: enhancing the percutaneous delivery and anti-psoriatic efficacy. Int J Pharm. 2017;528(1-2):511-523.

23. Lee SG, Kang JB, Kim SR, et al. Enhanced topical delivery of tacrolimus by a carbomer hydrogel formulation with transcutol P. Drug Dev Ind Pharm. 2016;42(10):1636-1642.

24. Goebel AS, Neubert RH, Wohlrab J. Dermal targeting of tacrolimus using colloidal carrier systems. Int J Pharm. 2011;404(1-2):159-168.

25. Savić V, Todosijević M, Ilić T, et al. Tacrolimus loaded biocompatible lecithin-based microemulsions with improved skin penetration: structure characterization and in vitro/in vivo performances. Int $J$ Pharm. 2017:529(1-2):491-505.

26. Li G, Fan Y, Fan C, et al. Tacrolimus-loaded ethosomes: physicochemical characterization and in vivo evaluation. Eur J Pharm Biopharm. 2012;82(1):49-57.

27. Pople PV, Singh KK. Targeting tacrolimus to deeper layers of skin with improved safety for treatment of atopic dermatitis. Int J Pharm. 2010;398(1-2):165-178.

28. Andrade LM, Silva LAD, Krawczyk-Santos AP, et al. Improved tacrolimus skin permeation by co-encapsulation with clobetasol in lipid nanoparticles: study of drug effects in lipid matrix by electron paramagnetic resonance. Eur J Pharm Biopharm. 2017;119:142-149.

29. Pople PV, Singh KK. Development and evaluation of colloidal modified nanolipid carrier: application to topical delivery of tacrolimus. Eur $J$ Pharm Biopharm. 2011;79(1):82-94.

30. Lapteva M, Mondon K, Möller M, Gurny R, Kalia YN. Polymer micelle nanocarriers for the cutaneous delivery of tacrolimus: a targeted approach for the treatment of psoriasis. Mol Pharm. 2014;11(9):2989-3001.

31. Mathes C, Melero A, Conrad P, et al. Nanocarriers for optimizing the balance between interfollicular permeation and follicular uptake of topically applied clobetasol to minimize adverse effects. $J$ Control Release. 2016;223:207-214.

32. Silva CO, Rijo P, Molpeceres J, et al. Polymeric nanoparticles modified with fatty acids encapsulating betamethasone for anti-inflammatory treatment. Int J Pharm. 2015;493(1-2):271-284.

33. Samad MB, Chhonker YS, Contreras J, et al. Developing polyaminebased peptide amphiphiles with tunable morphology and physicochemical properties. Macromol Biosci. 2017;17(8).

34. Hu B, Yuan Y, Yan Y, et al. Preparation and evaluation of a novel anticancer drug delivery carrier for 5 -fluorouracil using synthetic bolaamphiphile based on lysine as polar heads. Mater Sci Eng C Mater Biol Appl. 2017;75:637-645.

35. Raafat D, Sahl HG. Chitosan and its antimicrobial potential - a critical literature survey. Microb Biotechnol. 2009;2(2):186-201.

36. Zhang Z, Tsai PC, Ramezanli T, Michniak-Kohn BB. Ploymeric nanoparticles-based topical delivery systems for the treatment of dermatological diseases. Wiley Interdiscip Rev Nanomed Nanobiotechnol. 2013;5(3):205-218.

37. Al-Kassas R, Wen J, Cheng AE, Kim AM, Liu SSM, Yu J. Transdermal delivery of propranolol hydrochloride through chitosan nanoparticles dispersed in mucoadhesive gel. Carbohydr Polym. 2016;153: 176-186.

38. Je JY, Kim SK. Chitosan derivatives killed bacteria by disrupting the outer and inner membrane. J Agric Food Chem. 2006;54(18):6629-6633.

39. Hussain Z, Katas H, Amin MC, Kumulosasi E, Sahudin S. Antidermatitic perspective of hydrocortisone as chitosan nanocarriers: an ex vivo and in vivo assessment using an NC/Nga mouse model. J Pharm Sci. 2013;102(3):1063-1075.

40. Khalil SK, El-Feky GS, El-Banna ST, Khalil WA. Preparation and evaluation of warfarin- $\beta$-cyclodextrin loaded chitosan nanoparticles for transdermal delivery. Carbohydr Polym. 2012;90(3):1244-1253. 
41. Rasool AA, Hussain AA, Dittert LW. Solubility enhancement of some water-insoluble drugs in the presence of nicotinamide and related compounds. J Pharm Sci. 1991;80(4):387-393.

42. Lim LY, Go ML. Caffeine and nicotinamide enhances the aqueous solubility of the antimalarial agent halofantrine. Eur J Pharm Sci. 2000; 10(1):17-28.

43. Fan X, Chen J, Shen Q. Docetaxel-nicotinamide complex-loaded nanostructured lipid carriers for transdermal delivery. Int J Pharm. 2013; 458(2):296-304.

44. Huang Y, Zhang B, Gao Y, Zhang J, Shi L. Baicalein-nicotinamide cocrystal with enhanced solubility, dissolution, and oral bioavailability. J Pharm Sci. 2014;103(8):2330-2337.

45. Wohlrab J, Kreft D. Niacinamide - mechanisms of action and its topical use in dermatology. Skin Pharmacol Physiol. 2014;27(6):311-315.

46. Ungerstedt JS, Blömback M, Söderström T. Nicotinamide is a potent inhibitor of proinflammatory cytokines. Clin Exp Immunol. 2003;131(1): 48-52.

47. Rampino A, Borgogna M, Blasi P, Bellich B, Cesàro A. Chitosan nanoparticles: preparation, size evolution and stability. Int J Pharm. 2013; 455(1-2):219-228.

48. Gan Q, Wang T, Cochrane C, McCarron P. Modulation of surface charge, particle size and morphological properties of chitosan-TPP nanoparticles intended for gene delivery. Colloids Surf B Biointerfaces. 2005;44(2-3):65-73.

49. Lim SK, Kwon MS, Lee J, et al. Weissella cibaria WIKIM28 ameliorates atopic dermatitis-like skin lesions by inducing tolerogenic dendritic cells and regulatory T cells in BALB/c mice. Sci Rep. 2017;7:40040.

50. Leung DY, Hirsch RL, Schneider L, et al. Thymopentin therapy reduces the clinical severity of atopic dermatitis. J Allergy Clin Immunol. 1990; 85(5):927-933.

51. Hashimoto Y, Takaoka A, Sugimoto M, et al. Itch-associated scratching contributes to the development of dermatitis and hyperimmunoglobulinaemia E in NC/Nga mice. Exp Dermatol. 2011;20(10):820-825.

52. Abdel-Mottaleb MM, Moulari B, Beduneau A, Pellequer Y, Lamprecht A. Nanoparticles enhance therapeutic outcome in inflamed skin therapy. Eur J Pharm Biopharm. 2012;82(1):151-157.

53. Alvarez-Román R, Naik A, Kalia YN, Guy RH, Fessi H. Skin penetration and distribution of polymeric nanoparticles. J Control Release. 2004;99(1):53-62.

54. Jenning V, Gysler A, Schäfer-Korting M, Gohla SH. Vitamin A loaded solid lipid nanoparticles for topical use: occlusive properties and drug targeting to the upper skin. Eur J Pharm Biopharm. 2000;49(3):211-218.

55. Vogt A, Wischke C, Neffe AT, Ma N, Alexiev U, Lendlein A. Nanocarriers for drug delivery into and through the skin - do existing technologies match clinical challenges? J Control Release. 2016;242:3-15.
56. Prow TW, Grice JE, Lin LL, et al. Nanoparticles and microparticles for skin drug delivery. Adv Drug Deliv Rev. 2011;63(6):470-491.

57. Soma Y, Kashima M, Imaizumi A, Takahama H, Kawakami T, Mizoguchi M. Moisturizing effects of topical nicotinamide on atopic dry skin. Int J Dermatol. 2005;44(3):197-202.

58. Escudero-Góngora MM, Fernández-Peñas P. Nicotinamide: new indications in dermatology. Actas Dermosifiliogr. 2016;107(9): $777-778$.

59. Namazi MR. Nicotinamide in dermatology: a capsule summary. Int J Dermatol. 2007;46(12):1229-1231.

60. Chen AC, Martin AJ, Choy B, et al. A phase 3 randomized trial of nicotinamide for skin-cancer chemoprevention. $N$ Engl J Med. 2015; 373(17):1618-1626.

61. Mohammed D, Crowther JM, Matts PJ, Hadgraft J, Lane ME. Influence of niacinamide containing formulations on the molecular and biophysical properties of the stratum corneum. Int J Pharm. 2013;441(1-2): 192-201.

62. Offerta A, Bonina F, Gasparri F, Zanardi A, Micicche L, Puglia C. In vitro percutaneous absorption of niacinamide and phytosterols and in vivo evaluation of their effect on skin barrier recovery. Curr Drug Deliv. 2016;13(1):111-120.

63. Chen AC, Damian DL. Nicotinamide and the skin. Australas J Dermatol. 2014;55(3):169-175.

64. Chan CC, Liou CJ, Xu PY, et al. Effect of dehydroepiandrosterone on atopic dermatitis-like skin lesions induced by 1-chloro-2,4-dinitrobenzene in mouse. J Dermatol Sci. 2013;72(2):149-157.

65. Kang BK, Kim MJ, Kim KB, Ahn DH. In vivo and in vitro inhibitory activity of an ethanolic extract of Sargassum fulvellum and its component grasshopper ketone on atopic dermatitis. Int Immunopharmacol. 2016;40:176-183.

66. Tanno O, Ota Y, Kitamura N, Katsube T, Inoue S. Nicotinamide increases biosynthesis of ceramides as well as other stratum corneum lipids to improve the epidermal permeability barrier. Br J Dermatol. 2000; 143(3):524-531.

67. Greenstein RJ, Su L, Brown ST. Growth of M. avium subspecies paratuberculosis in culture is enhanced by nicotinic acid, nicotinamide, and $\alpha$ and $\beta$ nicotinamide adenine dinucleotide. Dig Dis Sci. 2011; 56(2):368-375

68. Moëll A, Skog O, Ahlin E, Korsgren O, Frisk G. Antiviral effect of nicotinamide on enterovirus-infected human islets in vitro: effect on virus replication and chemokine secretion. J Med Virol. 2009;81(6): 1082-1087.

69. Qi L, Xu Z, Jiang X, Hu C, Zou X. Preparation and antibacterial activity of chitosan nanoparticles. Carbohydr Res. 2004;3399(16): 2693-2700.
International Journal of Nanomedicine

\section{Publish your work in this journal}

The International Journal of Nanomedicine is an international, peerreviewed journal focusing on the application of nanotechnology in diagnostics, therapeutics, and drug delivery systems throughout the biomedical field. This journal is indexed on PubMed Central, MedLine, CAS, SciSearch ${ }^{\circledR}$, Current Contents ${ }^{\circledR} /$ Clinical Medicine,

\section{Dovepress}

Journal Citation Reports/Science Edition, EMBase, Scopus and the Elsevier Bibliographic databases. The manuscript management system is completely online and includes a very quick and fair peer-review system, which is all easy to use. Visit http://www.dovepress.com/ testimonials.php to read real quotes from published authors. 